PROCEEDINGS OF THE

AMERICAN MATHEMATICAL SOCIETY

Volume 130, Number 8, Pages 2189-2195

S 0002-9939(02)06334-7

Article electronically published on January 17, 2002

\title{
ON THE COMPLETENESS OF FACTOR RINGS
}

\author{
S. LOEPP AND C. ROTTHAUS
}

(Communicated by Wolmer V. Vasconselos)

\begin{abstract}
Let $T$ be a complete local domain containing the integers with maximal ideal $M$ such that $|T / M|$ is at least the cardinality of the real numbers. Let $p$ be a nonmaximal prime ideal of $T$ such that $T_{p}$ is a regular local ring. We construct an excellent local ring $A$ such that the completion of $A$ is $T$, the generic formal fiber of $A$ is local with maximal ideal $p$ and if $I$ is a nonzero ideal of $A$, then $A / I$ is complete.
\end{abstract}

\section{INTRODUCTION}

Let $A$ be a local ring with maximal ideal $M$ and $M$-adic completion $\hat{A}$. If $A$ is not complete, and has dimension at least two, the "usual" case is that there are many nonzero ideals $I$ of $A$ such that $A / I$ is not complete. In this paper, we construct rings that do not satisfy this "usual" condition. In other words, we are interested in noncomplete rings $A$ such that $A / I$ is complete for every nonzero ideal $I$ of $A$.

In fact, examples of such rings are known to exist. In [9], it is shown that noncomplete excellent regular local rings $R_{s}$ exist satisfying

$$
\operatorname{dim} R_{s}=s+1
$$

and for any nonzero ideal $I$ of $R_{s}$, the ring $R_{s} / I$ is complete. In addition, $R_{s}$ satisfies the following property: If $q$ is a nonzero prime ideal of $\hat{R}_{s}$, then $q \cap R_{s} \neq(0)$. In other words, the dimension of the generic formal fiber of $R_{s}$ is zero. (For definitions and background information on the formal fibers and generic formal fiber of an integral domain, see [8.) Also, in [1, S. Abhyankar, W. Heinzer, and S. Wiegand construct a two-dimensional normal local domain $S$ such that $S$ is not Henselian but $\frac{S}{P}$ is Henselian for every height one prime ideal $P$ of $S$. In fact, one can show (using Weierstrass Preparation) that $\frac{S}{I}$ is complete for every nonzero ideal $I$ of $S$. This ring $S$ satisfies the property that if $q$ is a nonzero prime ideal of $\hat{S}$, then $q \cap S \neq(0)$. So, once again, for this example, the dimension of the generic formal fiber is zero. As far as the authors know, these are the only known examples of noncomplete

Received by the editors June 28, 2000 and, in revised form, February 22, 2001.

2000 Mathematics Subject Classification. Primary 13J05, 13J10.

Key words and phrases. Local rings, completions, factor rings, excellent rings.

The first author appreciates the hospitality of Michigan State University, where this project was conducted, and is grateful for the support of the National Science Foundation via DMS \#9973069.

The second author thanks the National Science Foundation for their support via DMS \#980122. 
local rings $A$ of dimension at least two satisfying the property that $A / I$ is complete for every nonzero ideal $I$ of $A$. In light of this, we ask the natural question: Let $A$ be a local integral domain such that $A / I$ is complete for all nonzero ideals $I$ of $A$. Does it follow that the dimension of the generic formal fiber of $A$ is zero?

In this paper, we construct examples showing that the answer to this question is no. Let $T$ be a complete local domain with maximal ideal $M$ containing the integers and such that $|T / M|$ is at least the cardinality of the real numbers. Let $p$ be a nonmaximal prime ideal of $T$ such that $T_{p}$ is a regular local ring. We construct an excellent local ring $A$ such that the completion of $A$ is $T$, the generic formal fiber of $A$ is local with maximal ideal $p$ and if $I$ is a nonzero ideal of $A$, then $A / I$ is complete. So, given $n \geq 2$ and $0 \leq t<n$, we can construct an excellent local ring $A$ with $\operatorname{dim} A=n$ and $A / I$ complete for all nonzero ideals $I$ of $A$. In addition, if, for example, $T$ is a regular local ring, $p$ can be any nonmaximal prime ideal of $T$. Thus, by choosing the height of $p$ to be $t$, we can ensure that the dimension of the generic formal fiber of $A$ is $t$.

The fact that $A$ is a noncomplete excellent local ring possessing a local generic formal fiber is interesting. In [6], it is shown that a certain class of complete regular local rings can be realized as the completion of a noncomplete excellent local ring possessing a local generic formal fiber. Theorem 7 in this paper (thanks to suggestions by the referee) shows that a larger class of complete local domains can be realized as the completion of a noncomplete excellent local ring possessing a local generic formal fiber.

We note here that it can be important to know which ideals $I$ of a ring $A$ satisfy that $A / I$ is complete. For example, in [3], Heinzer, Rotthaus and Sally show that if "enough" prime ideas of $A$ satisfy this condition, then there is a one-to-one correspondence between the prime ideals in $\hat{A}$ that are maximal in the generic formal fiber of $A$ and certain birational extensions of $A$. (See 3 for details.)

All rings in this paper are commutative with unity. When we say a ring is local, Noetherian is implied. For a ring with exactly one maximal ideal that is not necessarily Noetherian, we will use the term quasi-local. When we write $(T, M)$ is a local ring, we mean that $T$ is a local ring with maximal ideal $M$. Following Matsumura in [8], we will use $\alpha(A)$ to denote the dimension of the generic formal fiber of a local integral domain $A$. Finally, we will use $c$ to denote the cardinality of the real numbers.

The construction of the ring $A$ in our theorem is simply a refinement of the construction used in [6]. We make use of the result found in [5] that if $(R, M \cap R)$ is a quasi-local subring of a complete local ring $(T, M)$, the map $R \longrightarrow T / M^{2}$ is onto and $I T \cap R=I$ for every finitely generated ideal $I$ of $R$, then $R$ is Noetherian and the natural homomorphism $\hat{R} \longrightarrow T$ is an isomorphism. Given a complete local domain $(T, M)$ and a prime ideal $p$ of $T$, both satisfying the conditions of our theorem, we start with the prime subring of $T$. We then build a chain of subrings of $T$ (starting with the prime subring of $T$ ) with each member of the chain satisfying some "nice" properties. These rings will be called $p$-subrings and defined later. Our ring $A$ will be the union of this chain of subrings. Clearly, then, we must choose our rings in the chain carefully. Specifically, we must ensure that $I T \cap A=I$ for every finitely generated ideal $I$ of $A$ and that $A \longrightarrow T / M^{2}$ is onto. These conditions will guarantee that the completion of $A$ is $T$. In addition, we must have that $p \cap A=(0)$ and if $q$ is a prime ideal of $T$ not contained in $p$, then $q \cap A \neq(0)$. Finally, we must choose the chain so that $A / I$ is complete for every nonzero ideal $I$ of $A$. 


\section{The COnstruction}

As discussed in the previous section, the ring $A$ of our theorem will be the union of a chain of subrings of $T$. We use the following proposition from [5] to guarantee that the completion of $A$ is, in fact, $T$.

Proposition 1. If $(R, M \cap R)$ is a quasi-local subring of a complete local ring $(T, M)$, the map $R \longrightarrow T / M^{2}$ is onto and $I T \cap R=I$ for every finitely generated ideal $I$ of $R$, then $R$ is Noetherian and the natural homomorphism $\hat{R} \longrightarrow T$ is an isomorphism.

The following definition is taken from 6]. In our construction, we will want to ensure that each ring in our chain satisfies the conditions needed to be a $p$-subring.

Definition. Let $(T, M)$ be a complete local ring and $(R, R \cap M)$ a quasi-local subring. Let $p \neq M$ be a prime ideal of $T$. Suppose:

(i) $|R| \leq \sup \left(\aleph_{0},|T / M|\right)$ with equality implying $T / M$ is countable,

(ii) $R \cap p=(0)$, and

(iii) $R \cap Q=(0)$ for every $Q \in \operatorname{Ass} T$.

Then we call $R$ a $p$-subring of $T$.

Condition (i) will be used so that $R$ is "small" compared to $T$. This will allow us to choose elements in $T$ that satisfy certain transcendental properties over $R$. Adjoining these elements to $R$ is the process we will use to build our chain of subrings. Condition (ii) is needed so that $p$ will be in the generic formal fiber of $A$. When $T$ is an integral domain, condition (iii) is trivial. However, all our results except for Theorem 7 hold when $T$ is not an integral domain and so we prove them as such. In this case, we will use condition (iii) to guarantee that $A$ will be an integral domain.

The following lemma is Lemma 2 from 4. Note that it is really a generalization of the prime avoidance lemma.

Lemma 2. Let $(T, M)$ be a local ring. Let $C \subset$ Spec $T$, let $I$ be an ideal such that $I \not \subset P$ for every $P \in C$, and let $D$ be a subset of $T$. Suppose $|C \times D|<|T / M|$. Then $I \not \subset \bigcup\{P+r \mid P \in C, r \in D\}$.

Recall that to use Proposition 1, we need the map $A \longrightarrow T / M^{2}$ to be onto. In addition, we want $A / I$ to be complete for every nonzero ideal $I$ of $A$. Note that if $T$ is, in fact, the completion of $A$ where $I$ is a nonzero ideal of $A$ and $A \longrightarrow T / I T$ is onto, then $A / I$ will be complete. To satisfy this condition and ensure that $A \longrightarrow T / M^{2}$ is onto, we will construct $A$ so that $A \longrightarrow T / J$ is onto for every ideal $J$ of $T$ that is not contained in the ideal $p$. It turns out that this condition will also help us show that the $\operatorname{ring} A$ we construct is excellent. If $J$ is an ideal of $T$ not contained in $p$, and $\bar{u} \in T / J$, we use the following lemma to construct an appropriate $p$-subring of $T$ that contains an element of $\bar{u}$. This lemma will be used later to guarantee that $A \longrightarrow T / J$ is onto.

Lemma 3. Let $(T, M)$ be a complete local ring with $|T / M| \geq c, p$ a nonmaximal prime ideal of $T$ and $J$ an ideal of $T$ with $J \not \subset p$. Suppose $p$ contains all associated prime ideals of $T$. Let $R$ be a p-subring of $T$ and $\bar{u} \in T / J$. Then there exists a p-subring $S$ of $T$ such that $R \subset S \subset T$ and $\bar{u}$ is in the image of the map $S \longrightarrow T / J$. Moreover, if $\bar{u}=\overline{0}$, then $S \cap J \neq(0)$. 
Proof. Let $C=$ Ass $T \cup\{p\}$ and suppose $P \in C$. Define $D_{(P)}$ to be a full set of coset representatives $t+P$ that make $(u+t)+P$ algebraic over $R$ as an element of $T / P$. Now, $\left|D_{(P)}\right|=\max \left(|R|, \aleph_{0}\right)$. As $|T / M| \geq c$, and $R$ is a $p$-subring, we have $|R|<|T / M|$. So, $\left|D_{(P)}\right|<|T / M|$. Note that by hypothesis, $J \not \subset P$ for every $P \in C$. Let $D=\bigcup_{P \in C} D_{(P)}$ and note that $|C \times D|<|T / M|$. Now, use Lemma 2 to find an element $x \in J$ such that $x \notin \bigcup\{r+P \mid r \in D$ and $P \in C\}$. We claim that $S=R[x+u]$ localized at $R[x+u] \cap M$ is the desired $p$-subring.

Since $|R|<|T / M|$ and $|S|=\max \left(|R|, \aleph_{0}\right)$, we have $|S|<|T / M|$. Hence, condition (i) of $p$-subrings is satisfied. Now, suppose that $P \in C$. Then if $f \in$ $R[u+x] \cap P$, we have

$$
f=r_{n}(u+x)^{n}+\cdots+r_{1}(u+x)+r_{0} \in P .
$$

So, $f \equiv 0$ modulo $P$. By the way $x$ was chosen, we have that $r_{i} \equiv 0$ modulo $P$ for every $i$. So, $r_{i} \in P \cap R=(0)$. Hence, $S \cap P=(0)$ and it follows that $S$ is a $p$-subring. Note that under the map $S \longrightarrow T / J, u+x$ is mapped to $u+J$, so $u+J$ is in the image of the map. Also, note that if $\bar{u}=\overline{0}$, then $u+x \in J$. Since $(u+x)+p$ is transcendental over $R$ as an element of $T / p$, we have $u+x \neq 0$. It follows that $S \cap J \neq(0)$.

Lemma 4 is Lemma 6 from [6]. It will be needed to ensure that $I T \cap A=I$ for every finitely generated ideal $I$ of $A$. This property, of course, is needed to apply Proposition 1 .

Lemma 4. Let $(T, M)$ be a complete local ring, $p$ a prime ideal of $T$, and $R$ a $p$-subring. Suppose $I$ is a finitely generated ideal of $R$ and $c \in I T \cap R$. Then there exists a p-subring $S$ of $T$ with $R \subset S \subset T$ and $c \in I S$.

Definition. Let $\Omega$ be a well-ordered set and $\alpha \in \Omega$. We define $\gamma(\alpha)=\sup \{\beta \in$ $\Omega \mid \beta<\alpha\}$.

In Lemma 5 , we construct a $p$-subring that simultaneously satisfies many of the properties we desire.

Lemma 5. Let $(T, M)$ be a complete local ring with $|T / M| \geq c$ and $p$ a nonmaximal prime ideal of $T$. Let $J$ be an ideal of $T$ with $J \not \subset p$ and suppose all associated prime ideals of $T$ are contained in $p$. Let $\bar{u} \in T / J$. Suppose $R$ is a p-subring of $T$. Then there exists a p-subring $S$ of $T$ such that:

(i) $R \subset S \subset T$,

(ii) if $\bar{u}=\overline{0}$, then $S \cap J \neq(0)$,

(iii) $u$ is in the image of the map $S \longrightarrow T / J$,

(iv) for every finitely generated ideal $I$ of $S$, we have $I T \cap S=I$.

Proof. Use Lemma 3 to find a $p$-subring $R_{0}$ such that $R \subset R_{0} \subset T$ and $\bar{u} \in$ Image $\left(R_{0} \longrightarrow T / J\right)$ and if $\bar{u}=\overline{0}$, then $R_{0} \cap J \neq(0)$. The rest of the proof follows the proof of Lemma 12 in [6], but the proof is not long and so we include it here.

We will construct $S$ to contain $R_{0}$, so conditions (i), (ii) and (iii) will follow automatically. Let

$$
\Omega=\left\{(I, c) \mid I \text { a finitely generated ideal of } R_{0} \text { and } c \in I T \cap R_{0}\right\} .
$$

Now, since $I$ can be $R_{0}$, we have $\left|R_{0}\right| \leq|\Omega|$, and $|\Omega| \leq\left|R_{0}\right|$ is clear since the number of finite subsets of $R_{0}$ is $\left|R_{0}\right|$. So, $|\Omega|=\left|R_{0}\right|$. Well-order $\Omega$ so that it does not have a maximal element and let 0 denote its initial element. Now, we 
will define a family of $p$-subrings. Begin with $R_{0}$. If $\gamma(\alpha) \neq \alpha$, and $\gamma(\alpha)=(I, c)$, then choose $R_{\alpha}$ to be the $p$-subring gotten from Lemma \so that $R_{\gamma(\alpha)} \subset R_{\alpha} \subset T$ and $c \in I R_{\alpha}$. If $\gamma(\alpha)=\alpha$, choose $R_{\alpha}=\bigcup_{\beta<\alpha} R_{\beta}$. Set $R_{1}=\bigcup R_{\alpha}$. Now, if $I$ is any finitely generated ideal of $R_{0}$, and $c \in I T \cap R_{0}$, then $(I, c)=\gamma(\alpha)$ for some $\alpha \in \Omega$. So, $c \in I R_{\alpha} \subset I R_{1}$. Thus, $I T \cap R_{0} \subset I R_{1}$. It is easy to verify that $R_{1}$ is a $p$-subring.

We repeat the process to obtain a $p$-subring extension $R_{2}$ of $R_{1}$ such that $I T \cap$ $R_{1} \subset I R_{2}$ for every finitely generated ideal $I$ of $R_{1}$. Continue to obtain an ascending chain $R_{0} \subset R_{1} \subset \cdots$ such that $I T \cap R_{n} \subset I R_{n+1}$ for every finitely generated ideal $I$ of $R_{n}$. Then, $S=\bigcup R_{i}$ is a $p$-subring. If $I$ is a finitely generated ideal of $S$, then some $R_{n}$ contains a generating set for $I$, say $y_{1}, \ldots, y_{k}$. If $c \in I T \cap S$, then $c \in R_{m}$ for some $m \geq n$. So, $c \in\left(y_{1}, \ldots, y_{k}\right) T \cap R_{m}$; therefore, $c \in\left(y_{1}, \ldots, y_{k}\right) R_{m+1} \subset I$. Thus, $I T \cap S=I$, so condition (iv) holds.

Finally, we can construct a ring $A$ satisfying all conditions of our theorem except that $A$ be excellent. Note that Lemma 6 provides nonexcellent examples of rings $A$ with a local generic formal fiber and such that $A / I$ is complete for every nonzero ideal $I$ of $A$. In a certain sense, though, the ring $A$ in Lemma [6] is "almost" excellent. Condition (ii) implies that at least in characteristic 0 , all formal fibers except the generic formal fiber are geometrically regular. So, the only obstacle to $A$ being excellent is that the generic formal fiber may not be geometrically regular.

Lemma 6. Let $(T, M)$ be a complete local ring with $|T / M| \geq c$ and such that no integer of $T$ is a zero-divisor. Let $p$ be a nonmaximal prime ideal of $T$ that contains all the associated prime ideals of $T$. Suppose that $p$ intersected with the prime subring of $T$ is the zero ideal. Then there exists a local domain A such that:

(i) $\hat{A}=T$.

(ii) If $P$ is a nonzero prime ideal of $A$, then $T \otimes_{A} k(P)$ is a field where $k(P)=$ $A_{p} / P A_{P}$.

(iii) The generic formal fiber of $A$ is local with $p$ the maximal ideal.

(iv) If $I$ is a nonzero ideal of $A$, then $A / I$ is complete.

Proof. Define

$$
\Omega=\{u+J \in T / J \mid J \text { is an ideal of } T \text { with } J \not \subset p\} .
$$

Since $T$ is Noetherian, if $J$ is an ideal of $T$, then $J$ is finitely generated. Hence, $\mid\{J$ ideal of $T \mid J \not \subset p\}|\leq| T \mid$. But since $|T / M| \geq c$, we have $|T|=|T / M|$. Also, $|T / M| \leq|T / J| \leq|T|$, and so $|T / J|=|T / M|$ for every ideal $J$ of $T$. It follows that $|\Omega|=|T / M|$. Well-order $\Omega$ so that each element has fewer than $|\Omega|$ predecessors. Let 0 denote the first element of $\Omega$. We define $R_{0}^{\prime}$ to be the prime subring of $T$ and $R_{0}$ to be $R_{0}^{\prime}$ localized at $R_{0}^{\prime} \cap M$. Note that $R_{0}$ is a $p$-subring.

We recursively define a family of $p$-subrings as follows. $R_{0}$ is already defined. Let $\lambda \in \Omega$ and assume $R_{\beta}$ has been defined for every $\beta<\lambda$. Then $\gamma(\lambda)=u+J$ for some ideal $J$ of $T$ with $J \not \subset p$. If $\gamma(\lambda)<\lambda$, use Lemma 5 to obtain a $p$-subring $R_{\lambda}$ so that $R_{\gamma(\lambda)} \subset R_{\lambda} \subset T, u+J \in \operatorname{Image}\left(R_{\lambda} \longrightarrow T / J\right)$ and for every finitely generated ideal $I$ of $R_{\lambda}$ we have $I T \cap R_{\lambda}=I$. Moreover, if $u+J=0+J$, we have $R_{\lambda} \cap J \neq(0)$. If $\gamma(\lambda)=\lambda$, define $R_{\lambda}=\bigcup_{\beta<\lambda} R_{\beta}$. Then, $R_{\lambda}$ is a $p$-subring for every $\lambda \in \Omega$. We claim $A=\bigcup_{\lambda \in \Omega} R_{\lambda}$ is the desired domain.

Now, as each $R_{\lambda}$ is a $p$-subring, we have $R_{\lambda} \cap p=(0)$. Hence, $A \cap p=(0)$. Also, if $J$ is an ideal of $T$ with $J \not \subset p$, then $0+J \in \Omega$. So, $\gamma(\lambda)=0+J$ for some $\lambda \in \Omega$ 
with $\gamma(\lambda)<\lambda$. By construction, $R_{\lambda} \cap J \neq(0)$. It follows that $J \cap A \neq(0)$. Hence, the generic formal fiber of $A$ is local with maximal ideal $p$.

We will now show that the completion of $A$ is $T$. To do this, we make use of Proposition[1. Note that as $p$ is not the maximal ideal of $T$, we have $M^{2} \not \subset p$. Hence, by the construction, the map $A \longrightarrow T / M^{2}$ is surjective. Let $I$ be a finitely generated ideal of $A$ with $I=\left(y_{1}, \ldots, y_{k}\right)$. Let $c \in I T \cap A$. Then $\left\{c, y_{1} \ldots, y_{k}\right\} \subset R_{\lambda}$ for some $\lambda \in \Omega$ with $\gamma(\lambda)<\lambda$. By construction, $\left(y_{1}, \ldots, y_{k}\right) T \cap R_{\lambda}=\left(y_{1}, \ldots, y_{k}\right) R_{\lambda}$. As $c \in\left(y_{1}, \ldots, y_{k}\right) T \cap R_{\lambda}$, we have $c \in\left(y_{1}, \ldots, y_{k}\right) R_{\lambda} \subset I$. Hence, $I T \cap A=I$. It follows by Proposition 1 that $A$ is Noetherian and the completion of $A$ is $T$.

Now suppose $I$ is a nonzero ideal of $A$. Let $J=I T$. If $J \subseteq p$, then $I \subseteq J \cap A \subseteq$ $p \cap A=(0)$, a contradiction. Hence, $J \not \subset p$. It follows by the construction that the map $A \longrightarrow T / J$ is surjective. Hence, $A / I \hookrightarrow T / J$ is surjective and hence $A / I$ is complete.

We now claim that if $J \not \subset p$, then $J=(A \cap J) T$. To see this, let $J \not \subset p$ and note that since $M \not \subset p$, we have $J M \not \subset p$. So, $A \longrightarrow T / J M$ is onto by our construction. Consider the $T$-modules $J, M$, and $(A \cap J) T$. We will show that $J=M J+(A \cap J) T$. Note that $M J+(A \cap J) T \subseteq J$ is clear. Now, let $x \in J$. Then since $A \longrightarrow T / J M$ is onto, there is an $a \in A$ such that $a+J M=x+J M$. So, $x=a+j m$ where $j \in J$ and $m \in M$. Also, $a=x-j m \in J$, so $a \in(A \cap J) T$. Hence, $x \in M J+(A \cap J) T$ and it follows that $M J+(A \cap J) T=J$. By Nakayama's Lemma, we have $J=(A \cap J) T$. So, our claim that if $J \not \subset p$, then $J=(A \cap J) T$ holds.

Now, suppose $P$ is a nonzero prime ideal of $A$ and $q$ is a prime ideal of $T$ such that $q \cap A=P$. Then $q \subseteq p$ implies that $q \cap A=p \cap A=(0)$, a contradiction. So, we must have that $q \not \subset p$. Hence, $q=(q \cap A) T=P T$ and it follows that the only prime ideal of $T$ that lies over $P$ is $P T$. Therefore, there is only one prime ideal in the ring $T \otimes_{A} k(P)$. Since $P T$ is a prime ideal of $T$, we must have that $T \otimes_{A} k(P)$ is a domain. It follows that $T \otimes_{A} k(P)$ is a field and hence property (ii) of the theorem holds. (In fact, one can show that $T \otimes_{A} k(P)$ is isomorphic to $k(P)$.)

It is interesting to note that we have also shown that there is a one-to-one correspondence between nonzero prime ideals of $A$ and prime ideals of $T$ that are not in the generic formal fiber of $A$.

Theorem [7 is the desired result.

Theorem 7. Let $(T, M)$ be a complete local domain containing the integers and such that $|T / M| \geq c$. Let $p$ be a nonmaximal prime ideal of $T$ such that $T_{p}$ is a regular local ring. Then there exists an excellent local domain $A$ such that the completion of $A$ is $T$, the generic formal fiber of $A$ is local with maximal ideal $p$ and if $I$ is a nonzero ideal of $A$, then $A / I$ is complete.

Proof. Use Lemma 6 to construct $A$. The Theorem is clear except for the fact that $A$ is excellent. Note that $T \otimes_{A} k((0)) \cong T_{p}$ is a regular local ring by assumption. By Lemma 6, the ring $T \otimes_{A} k(P)$ is a field. Since $T$ contains the integers, $T \otimes_{A} k(P)$ is a field of characteristic zero. Hence, it follows that $A$ is excellent.

We do not know if Theorem 7 holds when $T$ contains a finite field.

It is interesting to note that the proof of Lemma [6 shows that for the rings we constructed in Lemma [6] and Theorem 7, there is actually a one-to-one correspondence between nonzero prime ideals of $A$ and prime ideals of $T$ that are not in the generic formal fiber of $A$. 
Also, note in particular that the ring $A$ from Lemma 6 and Theorem 7 satisfies the following property: For all prime ideals $q$ of $T$ such that $p \subset q$, the ring $A /(q \cap A)$ is complete. Therefore, by Theorem 2.7 in [3], there is exactly one analytically irreducible normal local Noetherian domain $C$ that birationally dominates $A$ satisfying $\alpha(C)=0$ and satisfying $C / \mathbf{m} C$ is a finite $A$-module. (Here, $\mathbf{m}$ denotes the maximal ideal of $A$.)

In fact, one could modify the proofs in this paper by replacing the ideal $p$ with a countable set of nonmaximal, incomparable prime ideals of $T$. Doing this, one would see that all the results in this paper hold when $p$ is replaced with such a set. In particular, one could show that the following result holds: Let $(T, M)$ be a complete local domain containing the integers and such that $|T / M| \geq c$. Let $L$ be a countable set of nonmaximal incomparable prime ideals of $T$ such that $T_{p}$ is a regular local ring for all $p \in L$. Then there exists an excellent local domain $A$ such that the completion of $A$ is $T$, the maximal ideals in the generic formal fiber of $A$ is exactly the set $L$ and if $I$ is a nonzero ideal of $A$, then $A / I$ is complete. Then, by Theorem 2.7 in [3], there is a one-to-one correspondence between elements of $L$ and analytically irreducible normal local Noetherian domains $C$ that birationally dominate $A$, have $\alpha(C)=0$ and satisfy that $C / \mathbf{m} C$ is a finite $A$-module where $\mathbf{m}$ denotes the maximal ideal of $A$.

\section{ACKNOWLEDGEMENT}

We would like to thank the referee for many helpful suggestions. For example, in the paper we originally submitted, the conditions on the complete local ring in Theorem 7 were stronger. We are grateful to the referee for pointing out how to weaken these conditions.

\section{REFERENCES}

[1] S. Abhyankar, W. Heinzer, and S. Wiegand, On the Compositum of Two Power Series Rings, Proc. Amer. Math. Soc. 112 (1991), 629-636. MR 91j:13015

[2] A. Grothendieck and J. Dieudonné, Éléments de Géométrie Algébrique IV, Partie 2, Publ. Math. I.H.E.S. 24 (1965). MR 33:7330

[3] W. Heinzer, C. Rotthaus, and J. D. Sally, Formal fibers and birational extensions, Nagoya Math. J., 131 (1993), 1-38. MR 95a:13008

[4] R. Heitmann, Characterization of completions of unique factorization domains, Trans. Amer. Math. Soc, 337 (1993), 379-387. MR 93g:13006

[5] R. Heitmann, Completions of Local Rings with an Isolated Singularity, J. Algebra 163 (1994), 538-567. MR 95f:13032

[6] S. Loepp, Excellent Rings with Local Generic Formal Fibers, J. Algebra 201 (1998), 573-583. MR 99a:13013

[7] H. Matsumura, Commutative Ring Theory, Cambridge University Press, 1986. MR 88h:13001

[8] H. Matsumura, On the dimension of formal fibres of a local ring, Algebraic Geometry and Commutative Algebra in Honor of Masayoshi Nagata (Kinokuniya, Tokyo, 1987), 261-266. MR 90a:13027

[9] C. Rotthaus, On rings with low dimensional formal fibres, J. Pure and Applied Algebra, 71 (1991), 287-296. MR 92j:13006

Department of Mathematics and Statistics, Williams College, Williamstown, MasSACHUSETTS 01267

E-mail address: sloepp@williams.edu

Department of Mathematics, Michigan State University, East Lansing, Michigan 48824

E-mail address: rotthaus@math.msu.edu 